\title{
Spectroscopic Measurements of Impurity Ion Toroidal and Poloidal Flow Velocities and Their Dependence on Vertical Magnetic Field in QUEST Toroidal ECR Plasmas*)
}

\author{
Nao YONEDA, Taiichi SHIKAMA, Hideki ZUSHI ${ }^{1)}$, Kazuaki HANADA ${ }^{1)}$, \\ Akinobu FUJIKAWA, Takumi ONCHI ${ }^{1)}$, Kengoh KURODA ${ }^{1)}$, Kuniaki NII, \\ Masahiro HASUO, Makoto HASEGAWA ${ }^{1)}$, Hiroshi IDEI ${ }^{1)}$, Kazuo NAKAMURA ${ }^{1)}$, \\ Yoshihiko NAGASHIMA $^{1)}$, Aki HIGASHIJIMA ${ }^{1)}$ and Takahiro NAGATA ${ }^{1)}$ \\ Department of Mechanical Engineering and Science, Graduate School of Engineering, \\ Kyoto University, Kyoto 615-8540, Japan \\ 1) Research Institute for Applied Mechanics, Kyushu University, Fukuoka 816-8580, Japan
}

(Received 28 December 2017 / Accepted 16 April 2018)

\begin{abstract}
Toroidal electron cyclotron resonance (ECR) plasma is an ECR heated plasma in an open toroidal magnetic field. The plasma contains no toroidal current and is used for the pre-ionization and non-inductive startup of a tokamak plasma. To obtain a deeper understanding of the basic properties of the plasmas produced in the spherical tokamak QUEST (Q-shu University Experiment with steady-state Spherical Tokamak), we have developed an optical emission spectroscopy system with multiple viewing chords and have used it to measure the spatial distributions of the toroidal and poloidal flow velocities of $\mathrm{C}^{2+}$ ions. We compare the measured velocities with those calculated from the ion drift equations using the plasma parameters reported for a similar spherical tokamak, LATE (the Low Aspect Ratio Torus Experiment).
\end{abstract}

(C) 2018 The Japan Society of Plasma Science and Nuclear Fusion Research

Keywords: emission spectroscopy, flow measurement, non-inductive startup, electron cyclotron resonance (ECR) heated plasma, spatial distribution

DOI: $10.1585 /$ pfr.13.3402087

\section{Introduction}

Toroidal ECR plasma is an electron cyclotron resonance heated plasma in an open toroidal magnetic field $\left(B_{\varphi}\right)$. Understanding the basic properties of such a plasma is important, since it is used for the pre-ionization and noninductive startup of a tokamak plasma. Figure 1 shows a schematic illustration and a visible image of the plasma in QUEST. The plasma is vertically elongated along the res-

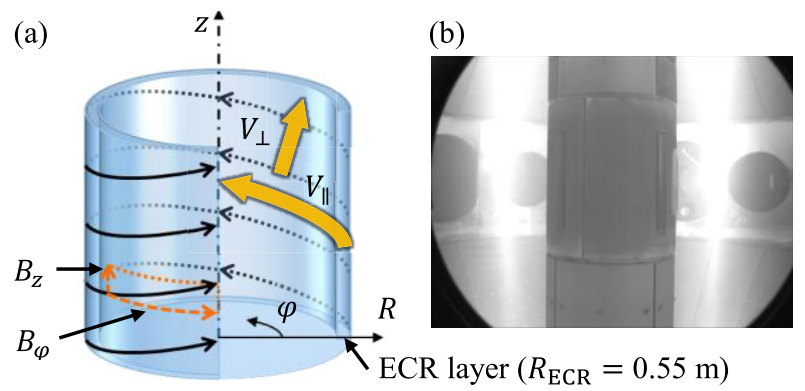

Fig. 1 (a) Schematic and (b) visible image of the toroidal ECR plasma in QUEST. The black arrows in (a) show the magnetic field lines.

author'se-mail: yoneda.nao.45m@st.kyoto-u.ac.jp

*) This article is based on the presentation at the 26th International Toki Conference (ITC26). onance layer, and a steep pressure gradient is produced in the radial direction.

In the LATE spherical tokamak, the two-dimensional spatial distributions of electron temperature $\left(T_{\mathrm{e}}\right)$ and density $\left(n_{\mathrm{e}}\right)$ and the space potential $\left(V_{\mathrm{s}}\right)$ have been measured in a poloidal section using a Langmuir probe $[1,2]$. In a weak vertical field $\left(B_{z}\right)$, the radial profiles of all parameters peak just outside the ECR layer, and the vertical profiles of $n_{\mathrm{e}}$ and $V_{\mathrm{s}}$ peak above the midplane [1]. From the measured plasma parameters, the radial and vertical ion flow velocities consist mainly of the radial and vertical components of the $E \times B$ and diamagnetic drift velocities, respectively, and the toroidal flow velocity consists mainly of the toroidal component of the Pfirsch-Schlüter flow velocity [3], which is given by

$$
v_{\mathrm{PS}}=-\frac{E_{R}}{B_{z}}+\frac{\nabla_{R} p_{\mathrm{i}}}{e n_{\mathrm{i}} B_{z}} .
$$

Here $E_{R}$ is the radial electric field, $\nabla_{R} p_{\mathrm{i}}$ is the radial gradient of the bulk ion pressure, $e$ is the elementary charge, and $n_{\mathrm{i}}$ is the $\mathrm{H}^{+}$ion density.

To confirm the generality of the results obtained in LATE, we have conducted measurements of the plasmas produced in the larger spherical tokamak QUEST $[4,5]$. Since it is difficult to measure the two-dimensional plasma parameters, we instead measured the spatial distributions 
of the toroidal and poloidal flow velocities of $\mathrm{C}^{2+}$ ions using optical emission spectroscopy, and we have investigated their consistency with the velocities predicted for LATE, assuming that the impurity ion flow velocity is in equilibrium with that of the bulk $\mathrm{H}^{+}$ions.

To obtain accurate measurements of the toroidal and poloidal flow velocities, we have developed a spectroscopy system with multiple viewing chords and high wavelength resolution.

\section{Experimental Setup}

QUEST is a medium-sized spherical tokamak. Its nominal major and minor radii are $0.64 \mathrm{~m}$ and $0.46 \mathrm{~m}$, respectively. A cylindrical $R \varphi z$ coordinate system is introduced, as shown in Fig. 1 (a). For our experiments, the applied toroidal magnetic field was $0.25 \mathrm{~T}$ at the plasma center, and its direction was counterclockwise as viewed from the top of the device. We used $8.2 \mathrm{GHz}, 40 \mathrm{~kW}$ microwaves for ECR heating. The position of the resonance layer on the midplane was $R_{\mathrm{ECR}}=0.55 \mathrm{~m}(0.29 \mathrm{~T})$. The vertical field was set to four different conditions $( \pm 10.4 \mathrm{mT}$ and $\pm 1.6 \mathrm{mT}$ ) at the intersection of the ECR layer and the midplane. Magnetic flux surfaces for a vertical field strength of $+1.6 \mathrm{mT}$ are shown in Fig. 2 (b). Table 1 summarizes the magnetic-field configurations in QUEST and in LATE [1].

A schematic of the spectroscopy system is shown in Fig. 3. It consists of a bifurcated optical-fiber bundle, and we denote the two ends as "bundle A" and "bundle B." Bundles A and B collect the emission from the plasmas and from a Th-Ne hollow cathode lamp (PerkinElmer Atomax), respectively. Each bundle consists of 16 optical fibers (Mitsubishi Cable Industries ST230D; 230 and $250 \mu \mathrm{m}$ core and clad diameters, respectively, and 0.2 NA) and an objective lens (Edmund Optics TS achromatic lens $9 \times 12$ VIS-NIR; $12 \mathrm{~mm}$ focal length and $9 \mathrm{~mm}$ diameter) .
Because of the limited size of the detector, we used 14 and 13 fibers for bundles A and B, respectively. The optical fibers of bundles A and B were aligned alternately at the entrance of the spectrometer (see Fig. 3). The collected light was transmitted to a Czerny-Turner spectrometer (Acton Research AM-510; $1 \mathrm{~m}$ focal length, F/8.7, and 1800 grooves/mm grating) through an astigmatism compensation system consisting of two concave mirrors (Tokyo Instruments; $0.3 \mathrm{~m}$ focal length). The spectra were recorded on a CCD (Andor Technology DU440-BU2; $2048 \times 512$ pixels and $13.5 \mu \mathrm{m}$ square pixels). The central wavelength of the spectrometer output was set at $468 \mathrm{~nm}$ to enable us to observe CIII emission lines $\left(2 \mathrm{~s} 3 \mathrm{~s}^{3} \mathrm{~S}-2 \mathrm{~s} 3 \mathrm{p}^{3} \mathrm{P}\right)$. We measured the spectra simultaneously over the wavelength range $461.3-474.5 \mathrm{~nm}$. The reciprocal linear dispersion was $6.4 \mathrm{pm} / \mathrm{pixel}$. The entrance slit width was set at $50 \mu \mathrm{m}$.

The ThI and NeI lines from the Th-Ne hollow cathode lamp were used as wavelength references. The positions of the recorded spectra on the CCD move slightly in the wavelength direction by up to 0.5 pixel with changes in room temperature, and this can produce a systematic ve-

Table 1 Differences between QUEST and LATE. $R_{\text {wall }}$ is the position of the inner wall of the vacuum vessel, $z_{\text {wall }}$ is the position of the top panel, and $P_{\mathrm{ECR} \text { heating }}$ is the microwave power used for ECR heating.

\begin{tabular}{|c|c|c|c|}
\hline & \multicolumn{2}{|c|}{ QUEST } & LATE \\
\hline$R_{\mathrm{ECR}}$ & \multicolumn{2}{|c|}{$0.55 \mathrm{~m}$} & $0.137 \mathrm{~m}$ \\
\hline$R_{\text {wall }}$ & \multicolumn{2}{|c|}{$1.3 \mathrm{~m}$} & $0.50 \mathrm{~m}$ \\
\hline$Z_{\text {wall }}$ & \multicolumn{2}{|c|}{$1.0 \mathrm{~m}$} & $0.30 \mathrm{~m}$ \\
\hline$B_{\varphi}\left(R=R_{\mathrm{ECR}}\right)$ & \multicolumn{2}{|c|}{$0.29 \mathrm{~T}$} & $0.0875 \mathrm{~T}$ \\
\hline$B_{z}$ & $10.4 \mathrm{mT}$ & $1.6 \mathrm{mT}$ & $0.6 \mathrm{mT}$ \\
\hline$B_{z} / B_{\varphi}$ & $\sim 1 / 30$ & $\sim 1 / 200$ & $\sim 1 / 150$ \\
\hline$P_{\mathrm{ECR} \text { heating }}$ & \multicolumn{2}{|c|}{$40 \mathrm{~kW}$} & $0.5,1.0,1.5 \mathrm{~kW}$ \\
\hline
\end{tabular}

(a)

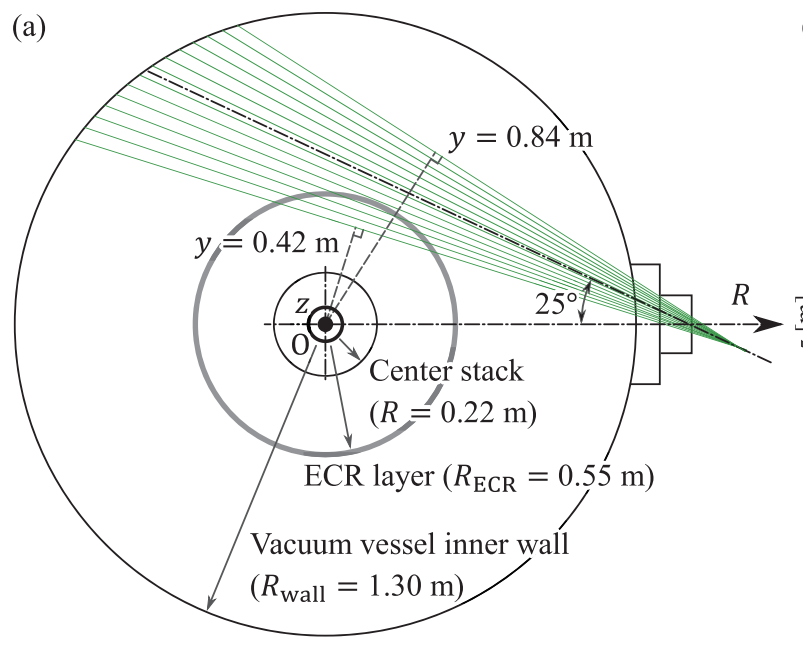

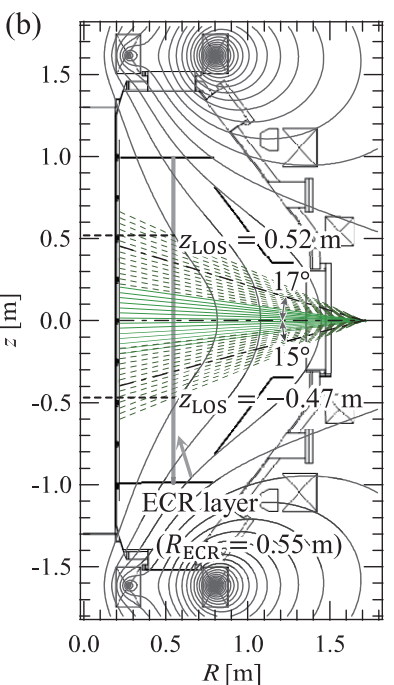

Fig. 2 Schematic of the experimental setups. (a) Toroidal setup (midplane). (b) Poloidal setups (poloidal section) with magnetic flux surfaces for $B_{z}=+1.6 \mathrm{mT}$.

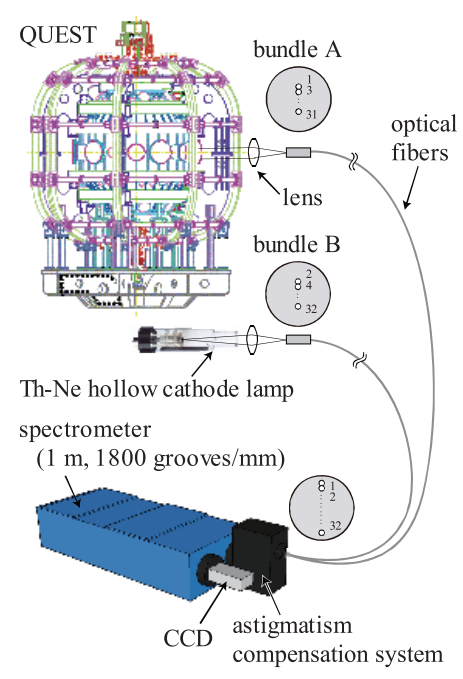

Fig. 3 Schematic of the spectroscopy system. 
locity error up to $2 \mathrm{~km} / \mathrm{s}$. Since we have confirmed that variations in room temperature cause no changes in relative positions of the spectra, we have calibrated the relative wavelengths using the spectra measured in advance from bundles $\mathrm{A}$ and $\mathrm{B}$. We calibrated the absolute wavelengths using the lamp spectra measured from bundle B during the times between discharges. This procedure reduced the systematic error to approximately $1.0 \mathrm{~km} / \mathrm{s}$. Using measurements from both bundles $\mathrm{A}$ and $\mathrm{B}$ decreased the error by approximately $0.3 \mathrm{~km} / \mathrm{s}$, in comparison with linear interpolation of the measurements from bundle B between discharges.

We regard a ThI emission line spectrum at 466.32026 $\mathrm{nm}$, which has negligibly small Doppler broadening, as the instrumental function. The full width of this line at half maximum was approximately $20 \mathrm{pm}$. The spectra measured on the inner and lower viewing chords, which are aligned on the midplane and the poloidal section respectively, are somewhat degraded because of their larger aberration.

Absolute calibration of the intensities of the measured spectra was accomplished using the continuum light from a standard tungsten-halogen lamp (Ushio Lighting) reflected by a diffuse plate (Labsphere SRS-99-020). From the Fresnel equations [6], the transmittance of the fused-quartz window was found to be approximately 0.92 .

We measured the flow velocities using the four setups described in Fig. 2 by changing the orientation of bundle $\mathrm{A}$ sequentially on a shot-by-shot basis. For the toroidal setup shown in Fig. 2 (a), the viewing chords were aligned on the midplane $(z=0)$. The perpendicular distances from the center of the vacuum vessel to the viewing chords, which we define as $y$, were $0.42-0.84 \mathrm{~m}$. Three poloidal setups are shown in Fig. 2 (b). The viewing chords were aligned on the poloidal section and then directed upward, horizontally, and downward. We adopted asymmetric setups for the upward and downward directions to avoid disturbance by a scanning probe located beneath the midplane. The $z$-positions of the viewing chords at the ECR layer, which

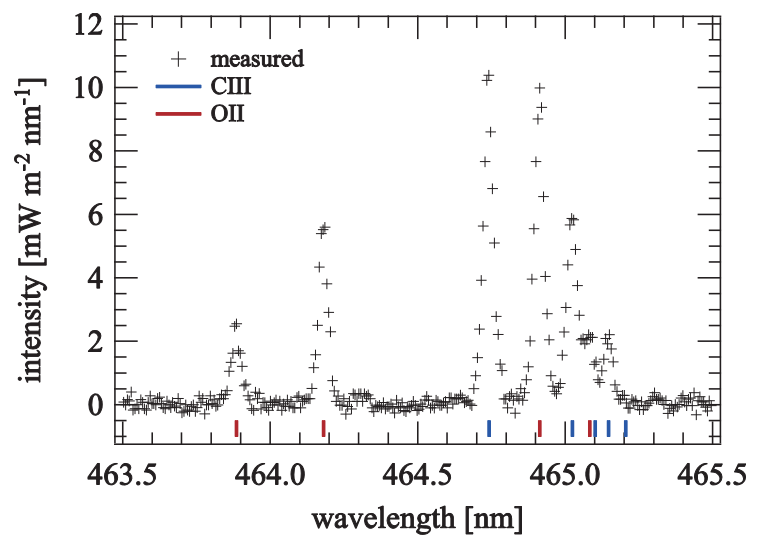

Fig. 4 The observed spectrum with $B_{z}=-10.4 \mathrm{mT}$, at $z_{\mathrm{LOS}}=$ $0.32 \mathrm{~m}$. are defined as $z_{\mathrm{LOS}}$, ranged from $-0.47 \mathrm{~m}$ to $0.52 \mathrm{~m}$.

A spectrum observed at $B_{z}=-10.4 \mathrm{mT}$ and $z_{\mathrm{LOS}}=$ $0.32 \mathrm{~m}$ is shown in Fig. 4. Among the several observed emission lines, we used a CIII emission line at $464.742 \mathrm{~nm}$ $\left(2 s 3 s^{3} S_{1}-2 s 3 p^{3} P_{2}\right)$ for the analysis. This line is isolated from adjacent lines and has a relatively large intensity. We evaluated the intensity and the temperatures and velocities of the ions-i.e., the Doppler broadening and shift-by approximating the line profile as a single Gaussian function. This means that the evaluated parameters are emissivityweighted averages along the viewing chords.

\section{Results and Discussion}

The measured velocities and intensities obtained with the toroidal and poloidal setups are shown in Figs. 5 and 6 , respectively. Negative velocities are directed toward the observer. The error bars include both random and systematic errors. The former originates mainly from the plasma and from shot noise, and the latter originates from the wavelength calibration. For the toroidal setup, the increased errors with increasing distances from the ECR layer to the viewing chords are due to decreases in the $S / N$ ratios of the spectra.

Since plasmas having the same values of parameter $B_{z} / B_{\varphi}$ are expected to be similar, we compared the spatial distribution of the intensity at $B_{z}= \pm 1.6 \mathrm{mT}\left(B_{z} / B_{\varphi} \sim\right.$ $1 / 200)$ in QUEST with that of the electron density at $B_{z}= \pm 0.6 \mathrm{mT}\left(B_{z} / B_{\varphi} \sim 1 / 150\right)$ in LATE. Under the assumption of coronal equilibrium, the intensity is approxi-

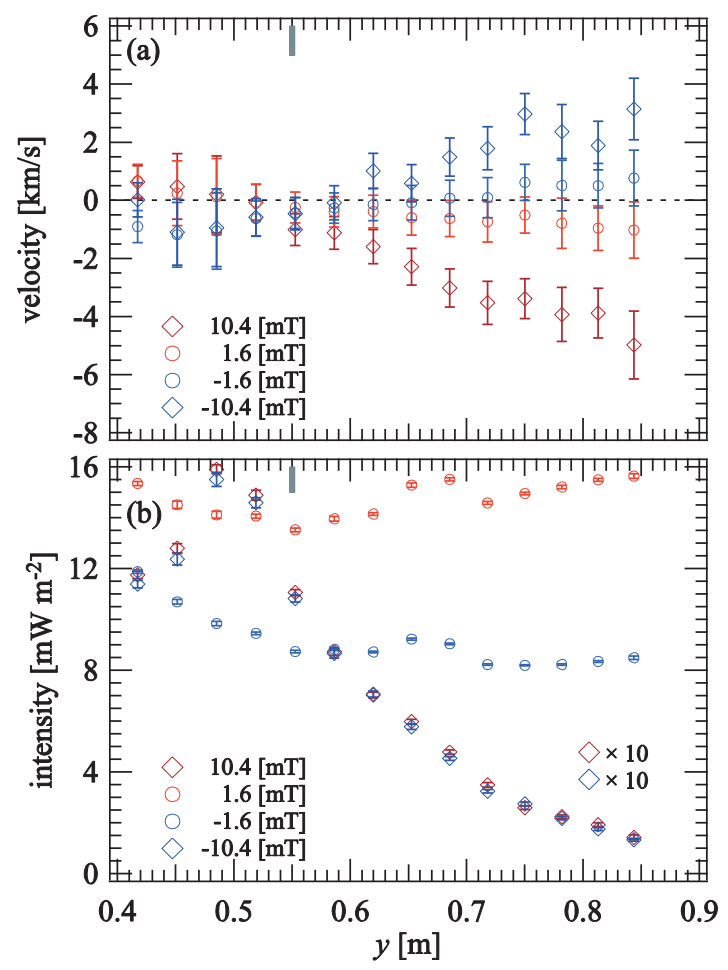

Fig. 5 (a) Flow velocities and (b) intensities measured using the toroidal setup. The gray lines indicate the ECR layer. 


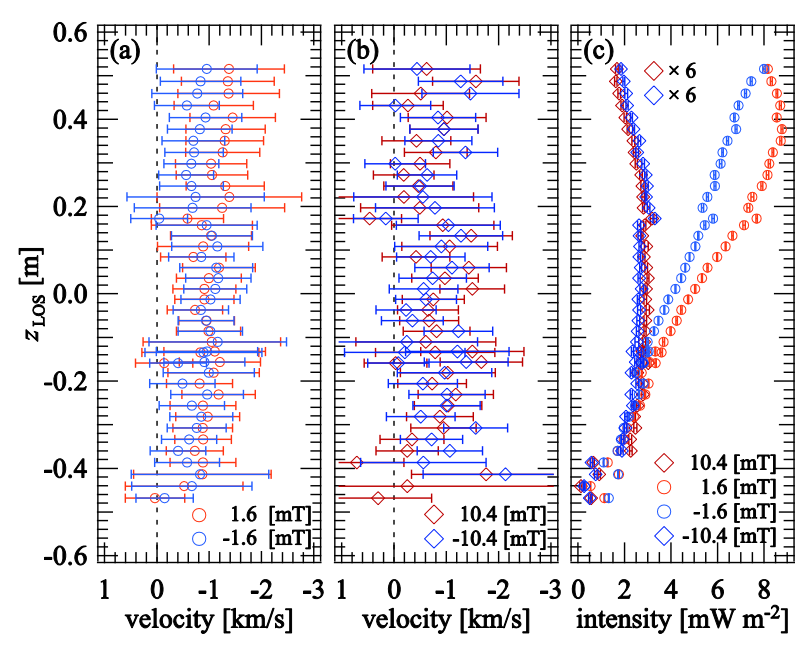

Fig. 6 Flow velocities with $B_{z}=(\mathrm{a}) \pm 1.6 \mathrm{mT}$, (b) $\pm 10.4 \mathrm{mT}$ and (c) intensities measured using the poloidal setups.

mately proportional to the product of the electron density and the ground-state $\mathrm{C}^{2+}$ ion density. Although the spatial distribution of the $\mathrm{C}^{2+}$ ion density is not known, the larger intensities for positive $B_{z}$ and the upward shift of the peak position upon switching $B_{z}$ from positive to negative are consistent with the results in LATE. We determined the ion temperature to be $\sim 10 \mathrm{eV}$ from the Doppler broadening of the spectra at $B_{z}= \pm 10.4 \mathrm{mT}$, which is similar to the electron temperature in LATE.

The flow velocities that we obtained are emissivityweighted averages along the viewing chords. The velocities measured with the toroidal setup are close to the local toroidal velocities at $y=R$ because the angle between the viewing chord and the velocity is smallest at this point [7]. For the poloidal setups, the measured flow velocities are projections of the radial and vertical velocities on the viewing chords. This means that the measured velocity is given by $v_{R} \cos \theta_{R}+v_{z} \cos \theta_{z}$, where $v_{R}$ and $v_{z}$ are the radial and vertical velocities, respectively, $\theta_{R}$ is the angle between the viewing chord and $v_{R}$, and $\theta_{z}$ is the angle between the viewing chord and $v_{z}$. Here, $\cos \theta_{R}$ is more than 0.9 , and $\left|\cos \theta_{z}\right|$ is less than 0.4 . The measured velocity is thus affected more by $v_{R}$ than by $v_{z}$ when $v_{R} \approx v_{z}$. In particular, for $B_{z}= \pm 10.4 \mathrm{mT}$, the emissivity is sharply peaked around the ECR layer, and its radial gradient is large so that the evaluated velocities approach the local velocities at the ECR layer.

The measured toroidal flow velocities in Fig. 5(a) have three features: (i) the flow direction changes around the ECR layer, (ii) the polarity of the flow velocity switches with that of $B_{z}$, and (iii) the magnitude of the flow velocity increases with the magnitude of $B_{z}$. The first feature is consistent with Eq. (1) because $E_{R}$ is directed away from the ECR layer and $\nabla_{R} p_{\mathrm{i}}$ is directed toward the layer. The second feature is also consistent. However, for the third feature, the observed $B_{z}$ dependence is opposite to that expected from Eq. (1). Additionally, the velocity calculated from Eq. (1) using quantities measured in LATE for
$B_{z} / B_{\varphi} \sim 1 / 150$ is on the order of $100 \mathrm{~km} / \mathrm{s}$, which is about two orders of magnitude larger than the measurements.

The velocities measured with the poloidal setups are approximately $1 \mathrm{~km} / \mathrm{s}$, as shown in Figs. 6 (a) and (b). The results are almost independent of $B_{z}$ and $z_{\text {LOS }}$. The former is consistent with the result obtained in LATE that the poloidal flow is mainly determined by $E_{R} \times B_{\varphi}$ and $E_{z} \times B_{\varphi}$ drifts. However, the latter implies a larger contribution of the $E_{z} \times B_{\varphi}$ drift to the measured velocities, since the vertical $E_{R} \times B_{\varphi}$ drift is predicted to be unidirectional, and the sign of $\cos \theta_{z}$ should change with that of $z_{\text {LOS }}$. The magnitude of the $E_{z} \times B_{\varphi}$ drift velocities expected from the results obtained in LATE is on the order of $1 \mathrm{~km} / \mathrm{s}$, which is consistent with the present results.

\section{Summary}

We have developed a spectroscopy system in QUEST that has a high wavelength resolution and multiple viewing chords, and we have used it to measure the $\mathrm{C}^{2+}$ ion flow velocities in toroidal ECR plasmas. When $B_{z} / B_{\varphi} \sim 1 / 200$, the spatial distributions of the intensity and the poloidal flow velocity suggest that the plasmas generated in QUEST and in LATE are similar, as expected. The radial and vertical flow velocities seem to be consistent with the $E_{z} \times B_{\varphi}$ and $E_{R} \times B_{\varphi}$ drifts, respectively, but the toroidal flow velocity cannot be explained by Pfirsch-Schlüter flow alone. This indicates the existence of other flow-driving mechanisms, such as ion pressure gradients or electric fields associated with the presheathes of the upper and lower divertor plates.

\section{Acknowledgments}

This work was supported in part by NIFS collaborative research program NIFS16KUTR114 and by collaborative research program of RIAM, Kyushu University 28FP3.

[1] S. Nishi, T. Sakabe, M. Uchida, H. Tanaka and T. Maekawa, Plasma Phys. Control. Fusion 52, 125004 (2010).

[2] K. Kuroda, M. Wada, M. Uchida, H. Tanaka and T. Maekawa, Plasma Phys. Control. Fusion 57, 075010 (2015).

[3] A.V. Chankin, D.P. Coster, N. Asakura, G. Corrigan, S.K. Erents, W. Fundamenski, H.M. Müller, R.A. Pitts, P.C. Stangeby and M. Wischmeier, Nucl. Fusion 47, 762 (2007).

[4] K. Hanada, K. Sato, H. Zushi, K. Nakamura, M. Sakamoto, H. Idei, M. Hasegawa, Y. Takasei, O. Mitarai, T. Maekawa et al., Plasma Fusion Res. 5, S1007 (2010).

[5] H. Idei, T. Kariya, T. Imai, K. Mishra, T. Onchi, O. Watanabe, H. Zushi, K. Hanada, J. Qian, A. Ejiri, M.M. Alam et al., Nucl. Fusion 57, 126045 (2017).

[6] E. Hecht, Optics, 4th ed., (Addison Wesley, San Francisco, 2002) pp.113-122.

[7] K. Mishra, H. Zushi, H. Idei, T. Onchi, M. Hasegawa, K. Hanada and QUEST Team, IEEE Trans. Plasma Sci. 44, 441 (2016). 